\section{BMI und Raucherstatus beeinflussen Revisionsrisiko nach Gelenkersatz nicht}

Burn E et al. The impact of BMI and smoking on risk of revision following knee and hip replacement surgery: evidence from routinely collected data. Osteoarthritis and Cartilage 2019; 27: 1294-1300

Übergewichtige und rauchende Patienten haben nach einem totalen Knie- (TKR) oder Hüftersatz (THR) unter anderem ein erhöhtes Risiko für thromboembolische Komplikationen. Ob die Patienten auch häufiger eine operative Revision benötigen, ist unklar. Eine aktuelle Studie untersucht den Zusammenhang zwischen Body-Mass-Index (BMI) und Raucherstatus und dem Risiko für eine Revision nach dem Gelenkersatz.
In der Studie wurden Daten von 10260 Patienten nach TKR und 10961 Patienten nach THR ausgewertet, die an einer rheumatoiden Arthritis (RA) und/oder an Arthrose litten. Die Krankenhausdaten zu den operativen Eingriffen wurden routinemäßig gesammelten Patientendaten aus der Primärversorgung gegenüberstellt. Die Daten stammten aus den Jahren 1997-2014.

Die Studienautoren nutzten parametrische Überlebensmodelle, um anhand des BMIs und des Raucherstatus (Raucher, Nichtraucher, ehemaliger Raucher) ein 10-Jahres Risiko für Revision und Sterblichkeit, sowie ein Lebenszeitrisiko für eine Revision zu errechnen. Dabei berücksichtigten sie neben BMI und Raucherstatus auch Alter, Geschlecht, Diagnose, Begleiterkrankungen und sozioökonomischen Status.

Aus der Auswertung ausgeschlossen wurden Patienten, bei denen ein gleichzeitiger bilateraler Gelenkersatz durchgeführt worden war. Litten Patienten an einer RA und Arthrose, wurde die RA als Leitdiagnose angenommen.

Die Patienten mit TKR waren im Schnitt 71 Jahre alt, weiblich, litten an einer Arthrose, hatten einen BMI von 30 und waren Nichtraucher. Die THR-Patienten waren im Schnitt 70, weiblich, litten an einer Arthrose, hatten einen BMI von 28 und waren Nichtraucher.

Die Autoren errechneten, dass eine Änderung des BMI von 25 auf 35 das 10-JahresRisiko für eine Revision nach TKR von 4,6\% (3,3-6,4\%) auf 3,7\% (2,6-5,1\%) verändert und das 10-Jahres-Risiko für eine Revision nach einer THR von 3,7 \% (2,8-5, $1 \%)$ auf $4,0 \%(2,8-5,7 \%)$.

Mit dem Rauchen anzufangen, so zeigen die Autoren, würde das 10-Jahres-Risiko für eine Revision nach einer TKR von 4,1\% $(3,1-5,5 \%)$ auf $2,8 \%(1,7-4,7 \%)$ verändern und von $3,8 \%(2,8-5,3 \%)$ auf $2,9 \%(1,9-$ $4,7 \%)$ nach einer THR.

Die Unterschiede sind statistisch nicht signifikant. Auch die geschätzten Lebenszeitrisiken hängen nicht signifikant vom BMI oder Raucherstatus ab.
FAZIT

Übergewicht und Rauchen erhöhen das Risiko für eine Revision nach Knie- und Hüftgelenkersatz bei rheumatoider Arthritis und Arthrose nicht.

Marisa Kurz M. Sc. B. A. München 